\title{
44214 - PAIN RELIEF AFTER CESAREAN SECTION: A PROSPECTIVE COHORT STUDY
}

\author{
Stephen Halpern, Sunnybrook \& Women's College HSC, Toronto, ON, Canada; \\ Jennifer Yee, Sunnybrook@Women's College Hospital; \\ Cyndy Oliver, Sunnybrook@Women's College Hospital; \\ P Angle, 76 Grenville St, Toronto, Ontario M5S1B2;
}

Introduction: Women should receive adequate analgesia after cesarean section. The Royal College of Anaesthetists1 has proposed standards for post $\mathrm{C} / \mathrm{S}$ pain relief, including the following: 1) $>90 \%$ women to have a worst pain score of $<3$ on a VAS of $0-10,2) 100 \%$ women to be prescribed NSAIDs, and 3) >90\% women to be satisfied with pain management. We sought to compare our practice with these standards.

Methods: After obtaining ethics approval, we recruited a convenience sample of 100 women. Questionnaires were administered via face-to-face interviews between postoperative days 2 and 4. Term, ASA I and II patients who had elective C/S under spinal anesthesia were included. We collected data related to the following: spinal morphine dose, analgesic consumption, worst pain scores, pain at rest and on movement, monitoring of respiratory rate and sedation level, side effects (pruritis, nausea and vomiting, drowsiness, constipation), and satisfaction with post $\mathrm{C} / \mathrm{S}$ pain management. Data were gathered from August to December, 2006. Descriptive statistics were used for group data. Non parametric tests were used for comparative data.

Results: 100 women were interviewed in hospital between 42 and $119 \mathrm{hrs}$ postoperatively. Demographic data are shown in the table. All women received a self-medication package upon transfer to the postpartum ward, including acetaminophen, ibuprofen, and docusate sodium. All patients who were did not have allergies to NSAIDS received them $(\mathrm{N}=98)$. The mean overall VAS worst pain score was $6.43+/-2.12$. No significant differences were seen in oxycodone consumption, worst pain scores, pain at rest and on movement as a function of spinal morphine dose. Patients who received $0.2 \mathrm{mg}$ of spinal morphine experienced more pruritus than those that received $0.15 \mathrm{mg}(\mathrm{p}=0.01) .94 \%(94 / 100)$ of women were satisfied or very satisfied with their pain management.

Discussion: The VAS pain scores were significantly higher than those recommended by the College, in spite of the administration of appropriate analgesia. However, maternal satisfaction with analgesia exceeded the recommendation. Our results suggest that the analgesic target, derived from the general surgery literature2, is not appropriate for obstetric patients possibly because of our poor understanding of pain measurement and the interplay between the pain experience and patient expectations.

References:

1 http://www.rcoa.ac.uk/docs/arb-section8.pdf Last accessed: Dec. 22, 2006. 2 BMJ 2002;305:1187-93. 


\begin{tabular}{|l|l|}
\hline \multicolumn{2}{|c|}{ Demographics } \\
\hline Mean Age yrs (sd) & $34(4)$ \\
\hline BMI (sd) & $29(6)$ \\
\hline Nullip/Multip & $41 / 59$ \\
\hline Singleton/Twins & $93 / 7$ \\
\hline Mg Dose of spinal morphine (n) & \\
\hline 0.15 & 65 \\
\hline 0.20 & 31 \\
\hline 0.30 & 4 \\
\hline
\end{tabular}

